1866 , vient de publier son $9^{m_{c}}$ rapport annuel, lequel embrasse la période du 3 août 1875 au 3 aout 1876. Il ressort de ce document que la dite fondation a secouru :

$1^{0}$ Dans l'administration centrale :

1088 invalides ou familles de soldats morts, par des dons continus, en y consacrant 161,268 marks, et 382 invalides ou familles de soldats morts, en une seule fois, avec 27,312 marks, soit en tout 188,580 marks ;

$2^{\circ}$ Dans les associations fliales:

521 invalides avec 32,109 marks, et 935 familles avec 72,048 marks, soit en tout 104,157 marks.

Durant les dix années d'existence de la fondation, il y a été dépensé en secours :

$1^{\circ}$ Dans l'administration centrale, 1,850,060 marks; - $2^{\circ}$ par les associations fliales, 1,133,378 marks; soit en tout 3,983,438 marks.

L'avoir actuel de la fondation s'élève encore :

$1^{\circ}$ Pour l'administration centrale, $\grave{a} 2,397,639$ marks, et $2^{\circ}$ pour les associations filiales, à 752,664 marks; soit en tout 3,150,303 marks.

\title{
BELGIQUE
}

AJOURNEMENT DE LA CONFËRENGE JE LA GROIX ROUGE

La lettre suivante a été adressée au Comité international par le Comité de Bruxelles :

Bruxelles, le 10 août 1876.

Messieurs,

Nous regrettons vivement de ne pas pouvoir donner suite au projet que nous avions formé de réunir à Bruxelles, pendant l'Exposition d'hygiène et de sauvetage, une Conférence intime des délégués des diverses sociétés de secours aux blessés. 
Nos intentions ne semblent pas avoir élé parfaitement comprises par tout le monde. Plusieurs des sociétés auxquelles nous nous étions adressés, ont donné à notre invitation une portée que nous n'avions nullement en vue.

Dans ces circonstances, nous pensons que le mieux est de renoncer à la réunion que nous avions projetée, et dont pourtant nous espérions quelque bien pour les malheureux.

Il nous reste à vous remercier de l'accueil bienveillant que vous aviez fait à notre proposition. Nous espérons pouvoir répéter ce remerciement de vive voix aux membres de votre association qui assiteront au Congrès d'hygiène et sauvetage. Nous serons extrêmement heureux de leur faire à cette occasion la réception la plus cordiale, et de témoigner ainsi de notre vif désir'd'entretenir, avec les sociétés similaires à la nôtre, les relations les plus amicales, dans l'intérêt de l'œuvre humanitaire à laquelle nous participons toụs.

Veuillez agréer, Messieurs, l'expression de nos sentiments les plus distingués.

Le Secrétaire,

F. Sigart.

Le Président,

Renard.

\section{L'EXPOSITION D'HYGIÈnE ET DE SAUVETAGE}

\section{A M. Gustave Moynier, président du Comité international de la Croix rouge.}

Mon cher Président,

Avant d'entrer dans les détails je vous dirai, d'une manière générale, qu'au milieu de cette exposition, si intéressante à tant d'égards et si bien réussie, les objets destinés aux secours pour les blessés occupent une place considérable et fort honorable.

L'Autriche et l'Allemagne y ont une série de wagons d'ambulance complétement aménagés et des voitures pour le transport des blessés. 